\title{
Interface homem-máquina para controle de processos de resfriamento com ar forçado visando à economia de energia
}

\author{
Man-machine interface for the control of cooling processes with forced-air aimed at energy savings
}

\author{
Bárbara Teruel $^{{ }^{*}}$ Paulo Silveira $^{\mathrm{II}}$ Filipe Marques $^{\mathrm{II}}$ Nelson Cappelli ${ }^{\mathrm{I}}$
}

RESUMO

Apresenta-se o desenvolvimento de um equipamento microprocessado, com saída de corrente, para controle da velocidade de rotação do motor do ventilador de sistemas de ar forçado, usando inversor de freqüência. Através de programação (software IHM.EXE), o usuário pode definir a massa a ser resfriada em quilogramas de produto. $O$ equipamento calcula, através de um polinômio previamente estabelecido e programável, a freqüência de operação do inversor, que corresponde a uma vazão de ar específica, dentro dos limites estabelecidos no projeto. O equipamento foi instalado num sistema de resfriamento com ar forçado, estimando-se, pelo cálculo da variação da potência útil mecânica, uma economia de energia da ordem de $82 \%$, com uma rotação equivalente a $56 \%$ da nominal do ventilador, proporcionando, desta forma, uma economia significativa no custo de operação do sistema.

Palavras-chave: massa de produto, vazão de ar, potência útil.

\section{ABSTRACT}

The development of micro processed equipment is presented, with current exit for control of the speed of rotation of the fan motor of the system of forced air, using investing of the frequency. Through programming (software IHM.EXE), the user can define the mass to be cooled, in kilograms of the product. The equipment calculates through a programmable polynomial previously defined, the frequency of operation of the investor which corresponds to air specific flux, within the limits of the project. The equipment was installed in a forcedair cooling system, being considered by the calculation in useful mechanical power, the energy savings is estimated at around $82 \%$ with a rotation equivalent to $56 \%$ of the fan nominal, thus providing significant savings in system operating costs.

Key words: product mass, air flow, useful power.

\section{INTRODUÇÃO}

O tema da eficiência energética é de grande importância nos tempos atuais. Particularmente no Brasil, o Programa Nacional de Conservação de Energia Elétrica (PROCEL) lançou a meta de que até 2015 deverão ser economizados 130 bilhões de kWh e, segundo dados publicados, a refrigeração é responsável pelo consumo de um terço de toda a energia produzida no mundo (PROCEL, 2006).

O Brasil, país de clima tropical, com temperaturas médias altas quase o ano todo, não pode prescindir de sistemas de refrigeração para a conservação de seus produtos hortícolas. Esta realidade, além das rígidas normas que os países importadores impõem e o apelo por alimento seguro, apontam para um cenário em que investimentos em sistemas de refrigeração tendem a crescer nos próximos anos.

Após a colheita de produtos hortícolas, o resfriamento rápido deve ser o primeiro passo a ser dado para a conservação destes. O resfriamento rápido permite a diminuição da temperatura, até patamares recomendados para cada tipo e variedade, contribuindo assim para o aumento da vida útil dos produtos. Por outro lado, as câmaras de armazenamento dimensionadas para as condições em que os produtos foram previamente resfriados terão uma carga térmica menor, o que reduz os custos fixos e variáveis. $\mathrm{O}$ resfriamento com ar forçado é um método recomendado a todos os produtos hortícolas, garantindo taxa de

\footnotetext{
IFaculdade de Engenharia Agrícola, Universidade Estadual de Campinas (Unicamp), CP 6011, 13083-970, Campinas, SP, Brasil. Email: barbarat@agr.unicamp.br.*Autor para correspondência.

IIT\&S Equipamentos Eletrônicos, São Carlos, SP, Brasil.
} 
resfriamento eficiente, desde que respeitado os limites de projetos estabelecidos (ASHRAE, 1994; FRASER \& OTTEN, 1992; BROSNAN \& WEN, 2001; KADER, 2002).

Vários trabalhos têm estudado a relação custo-benefício entre a vazão de ar, o tempo de resfriamento e a qualidade do produto resfriado, considerando-se que a vazão de ar deve estar entre 0,001 a $0,006 \mathrm{~m} 3 \mathrm{~s}^{-1}$ por kg de produto a resfriar. Vazões acima do recomendado podem provocar aumento da transferência de massa, o que se reflete em perdas de peso dos produtos (BAIRD et al., 1985; ARIFIN \& CHAU, 1988; TALBOT et al., 1992; FRASER \& OTTEN, 1992; BROSNAN \& WEN, 2001; KADER, 2002; CASTRO et al., 2005).

Os sistemas existentes atualmente funcionam de acordo com a vazão de ar pré-determinada no projeto em função da quantidade de produto a resfriar. Quando as condições reais diferem das considerações do projeto quanto à quantidade de produto a resfriar por vez, situação muito comum em propriedades agrícolas e unidades de beneficiamento, o ventilador continuará a fornecer a vazão nominal escolhida, alterando-se a relação custo-benefício do processo. Estas variações das condições de projeto propiciam, além da perda de qualidade do produto, aumento do consumo de energia acima do previsto, aumentando assim os custos de resfriamento (TERUEL et al., 2000; TERUEL et al., 2002).

Levando-se em conta o controle de velocidade de motores assíncronos trifásicos de corrente alternada e as características intrínsecas de alguns tipos de carga, pode-se economizar energia elétrica. No caso dos ventiladores, é possível ter economia de energia considerável, pois a curva de consumo de potência está em função do cubo da rotação, ou seja, para baixas rotações, o consumo de potência é baixo; porém, quando aumenta a rotação, a potência cresce em função do cubo da velocidade de rotação (TURCO et al., 2002; PROCEL, 2004).
Neste trabalho, apresenta-se o desenvolvimento de um equipamento microprocessado, com interface homem-máquina, IHM (saída de corrente 4 a $20 \mathrm{~mA}$ ), para o controle eficiente de sistemas de resfriamento com ar forçado, para trabalhar com vazões de ar variáveis em função da quantidade de produto a resfriar, mantendo-se a vazão de ar na faixa de valores recomendados, para garantir uma adequada relação custo-benefício.

\section{MATERIAL E MÉTODOS}

O sistema de resfriamento com ar forçado onde foi instalada a IHM forma parte de um protótipo experimental instalado no Laboratório de Termodinâmica e Energia da Faculdade de Engenharia Agrícola da Universidade Estadual de Campinas. Os sistemas comerciais podem possuir um ou mais ventiladores, posicionados na parte traseira dos paletes, embaixo do evaporador (Figura 1a). Os paletes contendo os produtos são cobertos por lonas para garantir que o ar entre pelas laterais das embalagens $\mathrm{e}$ escoe pelo leito de frutos, sendo então succionado e retornado ao evaporador, onde é resfriado (Figura 1b).

O sistema de resfriamento com ar forçado está composto por um ventilador com vazão de $3.960 \mathrm{~m}^{3} \mathrm{~h}^{-1}$, rotação nominal de $3.500 \mathrm{rpm}$, freqüência de $60 \mathrm{~Hz}$, potência de 3,73kW (5 HP). O sistema conta com um inversor de freqüência (220-330V; 28,8 A, 0-170Hz). O sistema de resfriamento está instalado numa câmara frigorífica, com sistema de refrigeração de compressão de vapor (R-22) e potência frigorífica de 4,88kW.

Inicialmente, calculou-se a vazão de ar necessária para resfriar a massa de produto numa faixa de 50 até $1.200 \mathrm{~kg}$, aplicando-se uma vazão de ar de $0,001 \mathrm{~m}^{3} \mathrm{~s}^{-1}$ por kg de produto resfriado. Com o valor da vazão de ar para cada condição, foi aplicada a Lei dos Ventiladores para o cálculo da rotação do eixo do motor para cada nova vazão, através da equação 1 :
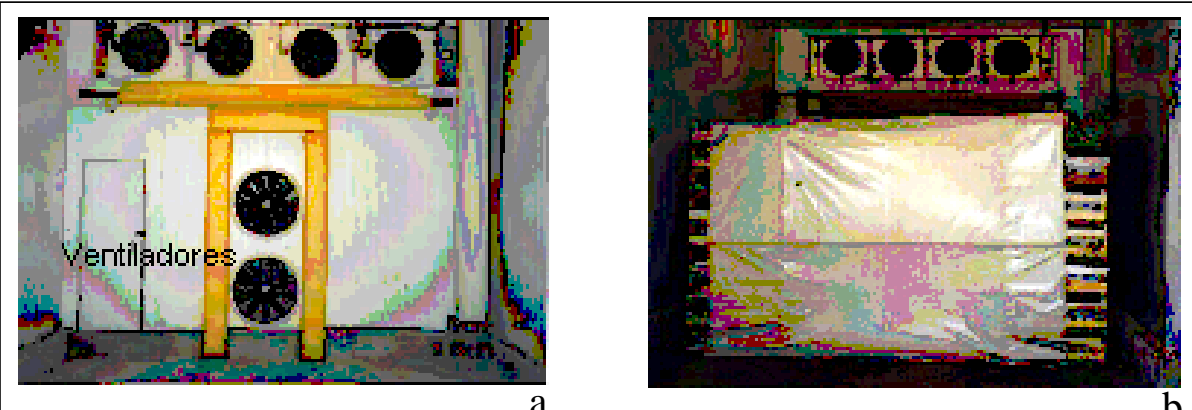

Figura 1 - (a) Posicionamento dos ventiladores em sistema de resfriamento com ar forçado. (b) Posicionamento dos paletes no túnel de ar forçado. 


$$
\frac{\mathrm{Q}_{1}}{\mathrm{Q}_{2}}=\frac{\mathrm{N}_{1}}{\mathrm{~N}_{2}}
$$

em que: $\mathrm{Q}_{1}$ é a vazão de ar nominal $\left(\mathrm{m}^{3} \mathrm{~s}^{-1}\right)$; $\mathrm{Q}_{2}$ é a vazão de ar para cada nova massa de produto $\left(\mathrm{m}^{3} \mathrm{~s}^{-1}\right)$; $\mathrm{N}_{1}$ é a rotação nominal (rpm) e $\mathrm{N}_{2}$ é a rotação que corresponde a cada nova massa de produto (rpm).

A velocidade de rotação do motor do ventilador também foi medida experimentalmente, com um tacômetro digital (0 a 15.000rpm), o qual foi posicionado no eixo do motor do ventilador, determinando-se posteriormente o desvio entre os valores calculados e medidos.

Para cada novo valor de rotação, $\mathrm{N}_{2}$, pôdese calcular o valor da freqüência correspondente (Equação 2). Os valores de freqüência foram também conferidos na tela digital do inversor de freqüência para cada condição.

$$
\mathrm{f}=\frac{\mathrm{np} \mathrm{N}}{120}
$$

em que: f é a freqüência (Hz) e np é o número de pólos do motor do ventilador $(\mathrm{np}=2)$.

Com os valores de massa, para o intervalo de 50 a $1.200 \mathrm{~kg}$ e os respectivos valores de freqüência, calculados com a equação 2, foi obtido um polinômio de 3 ardem, e determinadas as constantes introduzidas na programação da IHM (Equação 3):

$$
f=-A_{3}(m p)^{3}+A_{2}(m p)^{2}+A_{1}(m p)+A_{0}(3)
$$

em que: f é a freqüência calculada para cada valor de massa (Hz); mp é a massa de produto a ser resfriada (kg) e $A_{0}, A_{1}, A_{2}, A_{3}$ são as constantes do polinômio de $3^{\mathrm{a}}$ ordem

O valor da corrente corresponde à freqüência calculada, em função da massa e da vazão de ar, tendo sido determinada aplicando-se a equação 4 . $\mathrm{O}$ sinal de saída do inversor foi condicionado com um sinal de corrente entre 4 a $20 \mathrm{~mA}$, correspondente aos limites de freqüência máximo e mínimo estabelecidos (3 a $90 \mathrm{~Hz}$ ).

$$
\mathrm{I}=\mathrm{m} \cdot \mathrm{f}+\mathrm{b}
$$

em que: I é o valor de corrente em função da freqüência de operação (mA); m e b são as constantes do polinômio e f é a freqüência $(\mathrm{Hz})$.

O cálculo do coeficiente m é feito em função das freqüências máxima e mínima estabelecidas. A constante b é calculada em função do valor de m calculado e da freqüência mínima estabelecida.

Após o desenvolvimento do equipamento, implementou-se a IHM e o software de configuração (IHM.exe), testando-se a funcionalidade da primeira. Os cálculos da potência consumida foram realizados para cada novo valor de rotação, correspondente ao valor de massa do produto a resfriar, determinando-se uma estimativa da economia de energia que se poderia obter para as diferentes condições de operação (Equação 5):

em que: $\mathrm{P}_{1}$ é o valor da potência útil mecânica $(\mathrm{kW})$ e $\mathrm{P}_{2}$ é a potência útil mecânica $(\mathrm{kW})$ correspondente ao novo valor de rotação.

A estimativa apresentada diz respeito apenas à potência elétrica de entrada, correspondente à potência mecânica útil no eixo do motor do ventilador. Ou seja, não está sendo considerada a potência de entrada para suprir as perdas elétricas, o atrito, a ventilação e as perdas magnéticas associadas, além da potência necessária para suprir os campos magnéticos, que regem o princípio de funcionamento de máquinas elétricas.

\section{RESULTADOS E DISCUSSÂO}

As constantes determinadas para o ajuste da equação 3 foram: $\mathrm{A}_{0}=0,8223 ; \mathrm{A}_{1}=0,0419 ; \mathrm{A}_{2}=1 \mathrm{E}$ 05 e $A_{3}=-5 E-09$. Nota-se a existência de uma relação fortemente linear entre a freqüência e a massa que será resfriada, podendo-se considerar as constantes $\mathrm{A}_{2} \mathrm{e}$ $\mathrm{A}_{3}$ desprezíveis. Para o estudo realizado, uma relação linear é adequada e suficiente para se obter a curva de ajuste. No entanto, optou-se por um polinômio de $3^{a}$ ordem, permitindo-se maior flexibilidade ao sistema, visto que esse pode ser reprogramado para diferentes condições de operação, nas quais uma relação linear pode não ser precisa o suficiente. Esta reprogramação permite que a vazão de ar se mantenha dentro da faixa de valores recomendados para o processo de resfriamento do produto, garantindo a qualidade do produto e a economia de energia.

A diferença entre os valores de rotação medidos experimentalmente e os calculados por meio da equação 1 foi em média de 41,01士3,43rpm. Este erro pode ser considerado aceitável, podendo se adotar com segurança o cálculo de todos os parâmetros através das equações apresentadas.

Na figura 2a, apresenta-se a tela de programação do software IHM.EXE, escrito em Delphi 4.0, desenvolvido para a programação da IHM, na qual são introduzidos os valores das constantes do polinômio, as freqüências mínima e máxima de operação e indicada a porta serial para a comunicação com o computador. Se o usuário desejar, pode modificar os parâmetros, clicando em Programar Freqüências ou Programar Polinômio, quando as condições de operação foram modificadas.

O equipamento desenvolvido possui as seguintes características:

Ciência Rural, v.38, n.3, mai-jun, 2008. 


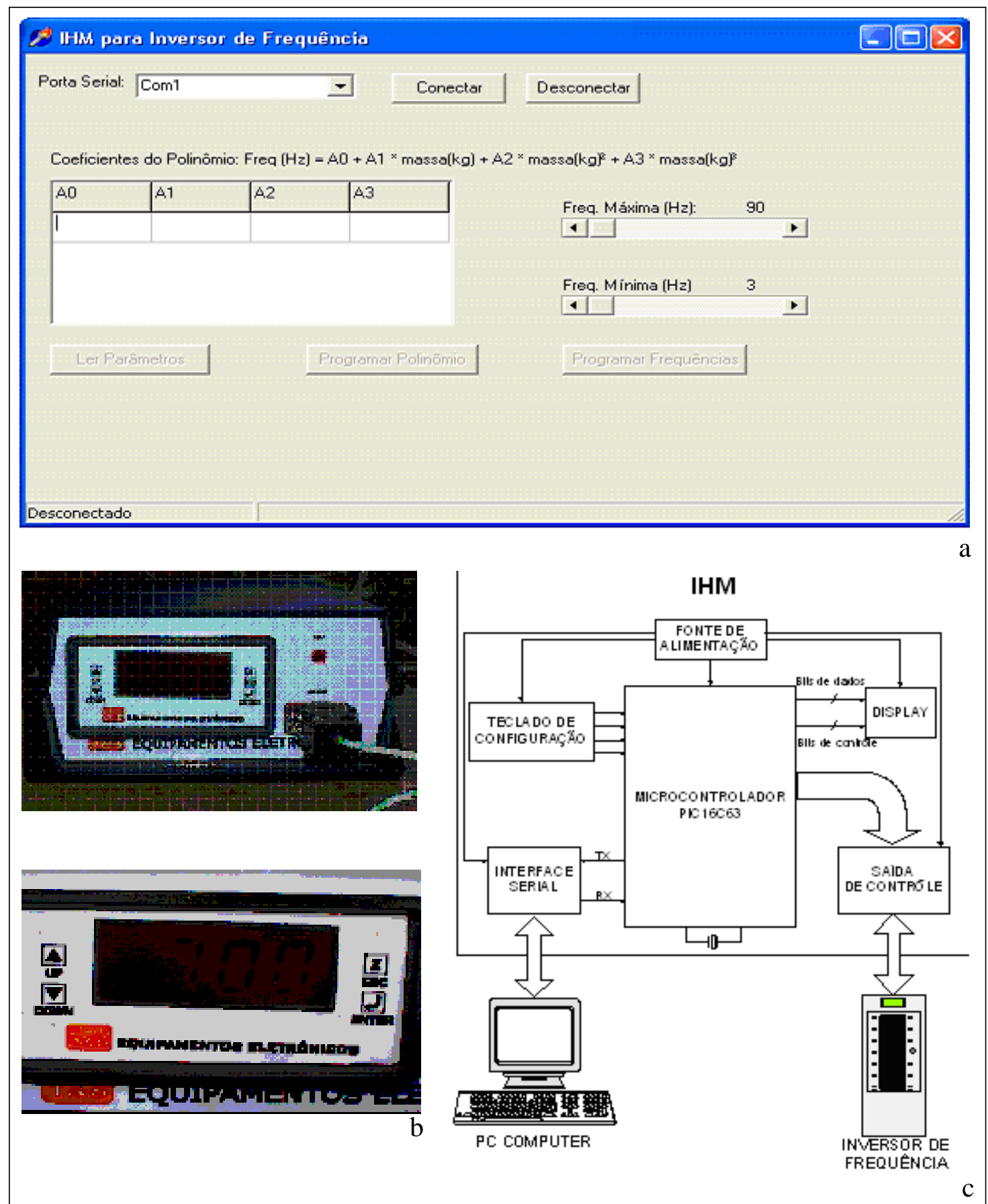

Figura 2 - (a) Tela de programação da Interface Homem-Máquina (IHM). (b) Vista frontal da IHM. (c) Diagrama de blocos da IHM.

- Microcontrolador modelo PIC16F876A, com memória ROM de 8kx 14 bits, RAM de 368 bytes; EEPROM de 256 bytes; interface serial RS-232, para reprogramação do microcontrolador; saída de corrente controlada de 4 a 20mA, impedância máxima de 500Ohms, para interface com inversor de freqüência; teclado com quatro teclas e display de sete segmentos com quatro dígitos, alimentação bivolt
110/220V selecionável, consumo máximo de 5W. Apresenta-se na figura $2 \mathrm{~b}$ o equipamento desenvolvido e, na figura 2c, o diagrama de blocos da IHM.

Estimativa preliminar da potência elétrica consumida A figura 3 apresenta as curvas que relacionam, em porcentagem, a potência consumida e a

Ciência Rural, v.38, n.3, mai-jun, 2008. 


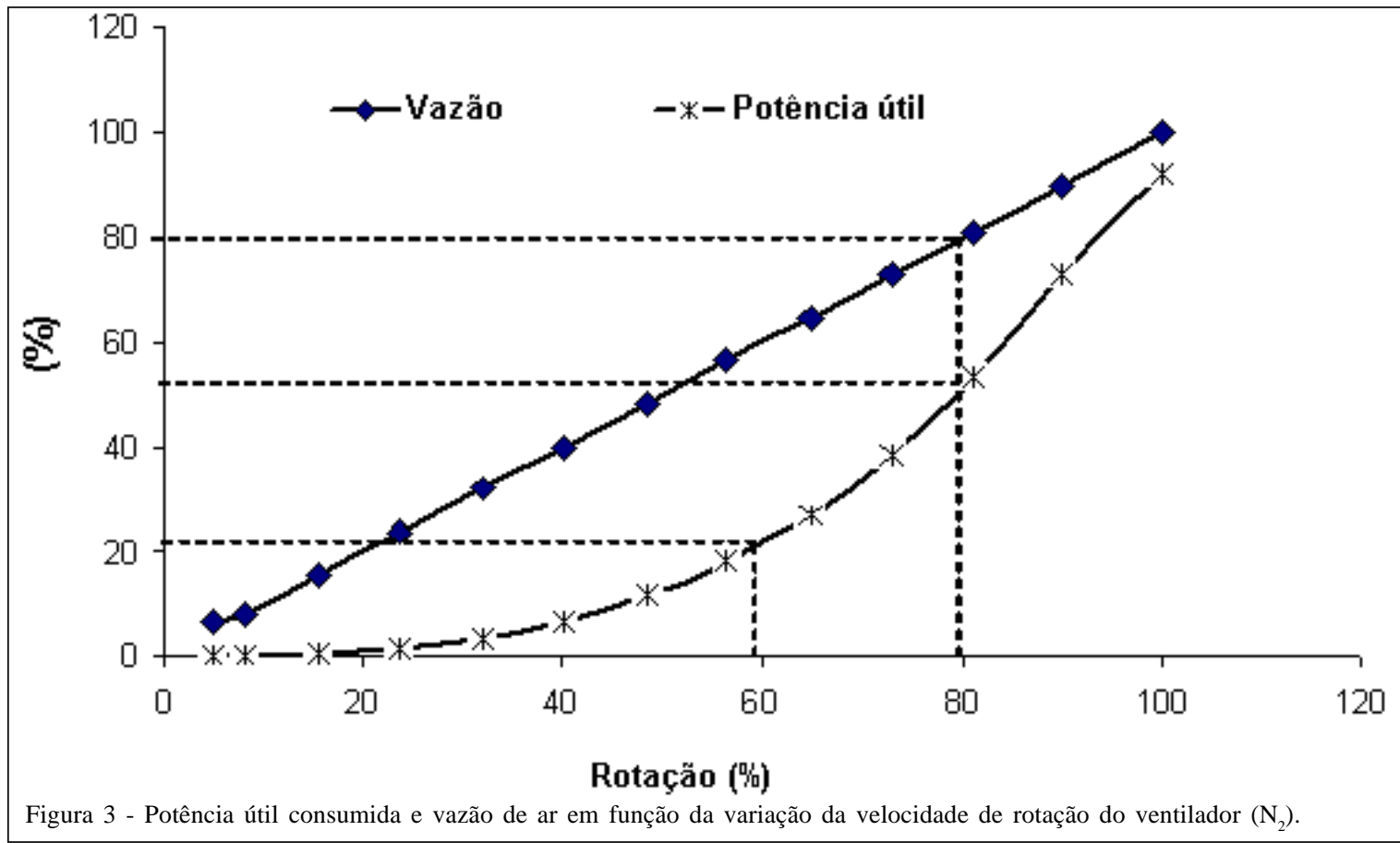

vazão em função da velocidade de rotação do motor do ventilador. Nota-se que, na rotação nominal do ventilador (100\%), o consumo de potência é máximo (100\%); no entanto, operando-se a $80 \%$ da rotação nominal, obtêm-se $80 \%$ da vazão equivalente a um consumo de potência útil de apenas 53\%. Este efeito é ainda mais significativo quando se opera a $60 \%$ da rotação nominal (60\% da vazão nominal), com um consumo de potência de apenas $22 \%$, que equivale a uma potência útil mecânica de $0,82 \mathrm{~kW}(1,1 \mathrm{HP})$. Através da IHM desenvolvida, poderão ser operados sistemas de resfriamento com ar forçado, de maneira tal a se obter economia de energia e qualidade do produto.

Resfriando-se $700 \mathrm{~kg}$ de produtos, com vazão de ar nominal e, com a vazão de ar alterada através da atuação da IHM no inversor de freqüência, haverá uma diferença da taxa de ar de 60\%. Para esta condição de operação, o motor do ventilador trabalhará aproximadamente a 56\% da rotação nominal (2.035rpm), significando uma redução da potência útil mecânica de $18 \%$, equivalente a $0,67 \mathrm{~kW}(0,89 \mathrm{HP})$ (Tabela 1$)$.

Como mostra a tabela 2, para 2.304h ano-1 de operação do sistema de resfriamento, a economia de energia elétrica poderia ser de até $82 \%$, equivalente ao consumo de potência elétrica que será transformada em potência útil mecânica.

Por sua vez, se a quantidade de produto a resfriar for inferior à considerada nos cálculos anteriores, por exemplo $100 \mathrm{~kg}$, a vazão de ar por kg de produto seria de $0,011 \mathrm{~m}^{3} \mathrm{~s}^{-1}$ (sem a IHM), valor 11 vezes maior que a vazão considerada no projeto $\left(0,001 \mathrm{~m}^{3} \mathrm{~s}^{-1}\right)$, trazendo aumento significativo do custo relativo ao consumo de energia elétrica, como discutido por BAIRD et al. (1985) e TERUEL et al. (2002).

O custo de resfriamento de produtos hortícolas, quando resfriados com ar forçado e diferentes vazões de ar (velocidade entre 0,3 e $2 \mathrm{~m} \mathrm{~s}^{-1}$ ), pode alcançar valores oscilando entre US\$ 0,50 a US\$

Tabela 1 - Variação dos parâmetros de operação do ventilador com e sem a IHM.

\begin{tabular}{lccc}
\hline Parâmetro & Condição $1^{*}$ & Condição 2** & Diferença*** $(\%)$ \\
\hline Quantidade de produto $(\mathrm{kg})$ & 700,00 & 700,00 & 0,0 \\
Vazão de ar nominal $\left(\mathrm{m}^{3} \mathrm{~h}^{-1}\right)$ & $3.960,00$ & $2.520,00$ & 36,4 \\
Taxa de $\mathrm{ar}\left(\mathrm{m}^{3} \mathrm{~s}^{-1} \mathrm{~kg} \mathrm{produto}^{-1}\right)$ & 0,0016 & 0,0010 & 37,5 \\
Rotação $(\mathrm{rpm})$ & $3.500,00$ & $2.035,00$ & 42,0 \\
Potência útil $(\mathrm{kW})$ & 3,73 & 3,06 & 18,0 \\
\hline
\end{tabular}

*Sem a Interface Homem Máquina (IHM); **com a IHM; *** Diferença entre as condições 1 e 2.

Ciência Rural, v.38, n.3, mai-jun, 2008. 
Tabela 2 - Estimativa de custos de energia elétrica consumida.

\begin{tabular}{|c|c|c|c|}
\hline Parâmetro & Condição $1^{\mathrm{a}}$ & Condição $2^{\mathrm{b}}$ & Diferença $^{\mathrm{c}}(\%)$ \\
\hline Tempo de operação (h ano $\left.{ }^{-1}\right)$ & $2.304,00 *$ & $2.304,00$ & - \\
\hline Custo de energia ( $\mathrm{R} \$ \mathrm{kWh}^{-1}$ ) & $0,25 * *$ & 0,25 & \\
\hline Conta de energia (R\$) [U\$] & $2.148,48[954,88]^{* * *}$ & $385,92[171,52]$ & 82,0 \\
\hline Diferença (1 e 2) (R\$) [U\$] & \multicolumn{2}{|c|}{$1.762,08[783,14]$} & \\
\hline
\end{tabular}

* 8 h dia $^{-1}, 6$ dias mês ${ }^{-1}, 12$ meses ano ${ }^{-1}$;* Custo da energia na área rural do Brasil, referência da Companhia Paulista de Força e Luz do

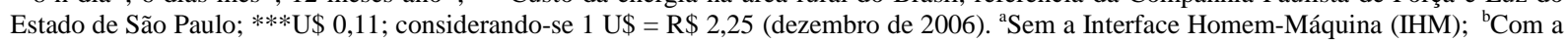
IHM; ' Diferença entre as condições 1 e 2.

0,95 por kilograma de produto (BAIRD et al., 1985). TERUEL et al. (2002) obtiveram valores de custo de resfriamento de $\mathrm{R} \$ 0,27$ a $\mathrm{R} \$ 0,41$ por caixa de laranja resfriada com ar forçado, sendo que o custo de energia elétrica representou até $12 \%$ do valor de custo total.

O equipamento foi desenvolvido pela empresa T\&S Equipamentos Eletrônicos. Incorporandose o inversor de freqüência, o custo de fabricação foi de $\mathrm{R} \$ 3.120,00$ (U\$ 1.386,66), que poderá ser absorvido pelo produtor agrícola em menos de dois anos, dependendo de cada caso em específico.

\section{CONCLUSÕES}

A IHM desenvolvida é de fácil manuseio, instalação e operação. O usuário pode selecionar, por meio de teclas, a quantidade de produto a ser resfriada e, após esta programação, o motor do ventilador trabalhará em uma nova condição, melhorando significativamente a eficiência no uso da energia elétrica, refletindo-se no custo de resfriamento.

Além disso, pode ser utilizada para diferentes condições de operação, reprogramando-se os parâmetros iniciais, mantendo-os dentro das recomendações de operação que vão garantir a qualidade do produto. Por fim, pode ainda ser adaptada a sistemas de resfriamento com ar forçado em operação em propriedades agrícolas e unidades de beneficiamento, trazendo benefícios para os produtores agrícolas.

\section{AGRADECIMENTOS}

À FAPESP (Fundação de Amparo à Pesquisa do Estado de São Paulo), pelo suporte financeiro. Ao Laboratório de Termodinâmica e Energia e ao Laboratório de Instrumentação e Controle, da Faculdade de Engenharia Agrícola da Universidade Estadual de Campinas. Ao Engenheiro Ernesto Barrientos, pela valiosa colaboração.

\section{REFERÊNCIAS}

ASHRAE. Systems and applications. Methods of precooling of fruits, vegetables and flowers. Atlanta: (American Society of Heating, Refrigerating and Air-Conditioning Engineers). 1994. cap.10, p.1-10.
ARIFIN, B.B.; CHAU, K.V. Cooling of strawberries in cartons with new vent hole designs. ASHRAE Transactions, v.94, p.1415-1426, 1988.

BAIRD, C.D. et al. An engineering/economic model for evaluating forced-air cooling systems for fruits and vegetables. Refrigeration Science and Technology. France: Institute International of Refrigeration, 1985. p.259-266.

BROSNAN, T.; WEN, S.D. Precooling techniques and applications for horticultural products- a review. International Journal of Refrigeration, v.24, p.154-170, 2001.

CASTRO, L.R et al. Effect of container openings and airflow rate on energy required for forced-air cooling of horticultural produce. Canadian Biosystem Engineering, v.21, p.3.13.9, 2005.

FRASER H.; OTTEN, L. Predicting 7/8 cooling times for peaches by comparing heat transfer modelling and field measurement methods. St. Joseph: (American Society of Agricultural Engineers) ASAE, 1992. 10p. (Paper n. 926016).

KADER, A.A. Postharvest technology of horticultural crops. 3.ed. Davis: Division of Agriculture and Natural Resources, University of California, 2002. 295p, n.3311.

PROCEL, Programa Nacional de Conservação de Energia Elétrica. Capturado em 2006. On line. Disponível em: http:/ /www.eletrobras.com/elb/procel/main.asp.

PROCEL Indústria- Eficiência Energética Industrial. Motor elétrico. Guia avançado. Brasília: PROCEL, 2004. 170p.

TALBOT, M.T. et al. Improving forced -air cooler performance. Florida: Florida Cooperative Extension Service, Institute of Food and Agricultural Sciences, University of Florida. 1992. 9p. (Circular AE 108).

TERUEL M.B. et al. Curvas de enfriamiento de naranjas refrigeradas en un sistema con aire forzado. Revista Información Tecnológica, CIT, v.11, p.25-29, 2000.

TERUEL, M.B. et al. Avaliação preliminar dos custos de resfriamento de laranja in natura. Revista Brasileira de Fruticultura, SP, v.24, p.86-90, 2002.

TURCO, J.E.P. et al. Consumo e custo de energia elétrica em equipamentos utilizados em galpão de frangos de corte. Revista Brasileira de Engenharia Agrícola e Ambiental. v.6, n.3, p.519-522, 2002. 